\title{
High-Order Simulation of Induced Disturbance in a Mach 6 Boundary Layer
}

\author{
Harold L. Atkins* \\ NASA Langley Research Center, Hampton, VA 23681-2199
}

\begin{abstract}
Simulations of the early evolution of induced unstable disturbances in a Mach 6 hypersonic boundary layer are presented for the purposes of validating a high-order discontinuous Galerkin Navier-Stokes simulation code. The simulations are modeled after experiments performed in the Boeing/AFOSR Mach-6 Quiet Tunnel at Purdue University. In these experiments, a forcing mechanism was employed to induce reproducible disturbances in a hypersonic boundary layer providing for the controlled study of the growth and breakdown of these disturbances into turbulent spots. Simulations revealed that the form and strength of the excitation can greatly influence the growth of the disturbance. In particular, at large forcing amplitude, the simulated forcing produces large advecting transients that appear to enhance the growth of the wave packet, relative to that of low amplitude forcing. Simulation results with large amplitude forcing agree well with experimental results while results from low amplitude forcing agree with linear stability theory.
\end{abstract}

\section{Introduction}

Experiments recently performed in the Boeing/AFOSR Mach-6 Quiet Tunnel (BAM6QT) at Purdue University ${ }^{1-3}$ studied the growth and breakdown of disturbances in a well controlled environment. This experiment was chosen as a validation case for a high-order three-dimensional Navier-Stokes simulation code based on the discontinuous Galerkin (DG) methodology. Prior work validating a two-dimensional variant of this code demonstrated super-convergence of unsteady viscous flow in the wake of a cylinder. ${ }^{4}$ Some recent work to improve robustness of the DG method in the presence of shocks is described in Ref. 5. That work also describes some preliminary simulations that employed an axisymmetric variant of the current code.

In the experiment, illustrated in Fig. 1 taken from Ref. 1, an electrode induces a disturbance that grows quickly into a nonlinear turbulent spot. Pressure is measured on the tunnel wall at several points downstream of the electrode. Typical ensemble-averaged results from the Purdue experiment are shown in Fig. 1(c). When operated in quiet flow mode, BAM6QT produces a relatively thick laminar boundary layer with a long transition region that enables accurate and detailed measurements with the available pressure sensors. Ideally, a study of naturally occurring disturbances would be preferred. However, the naturally occurring disturbances form in random locations relative to the pressure sensors and vary in strength making meaningful measurement difficult. The experiment employs an electrode forcing mechanism resulting in the production of consistent and repeatable wave packets.

In early experimental work ${ }^{1,2}$ the electrode was thought to produce a spark, presumably of very short duration; and the electrode was referred to as a "spark perturber." Guided solely by pressure measurements just downstream of the electrode and the assumption of a forcing of short duration, early axis-symmetric simulations ${ }^{5}$ attempted to reverse engineer a numerical forcing that would produce a similar downstream disturbance. However, the short duration of the forcing required an extremely large amplitude to produce any measurable disturbance. The large amplitude forcing posed a challenge to the robustness of the numerical simulation method, which would fail during the initial transient phase if the amplitude was too large. Efforts to improve the robustness of the method in the presence of shock waves ${ }^{5}$ were moderately successful, allowing

* Senior Research Scientist, Computational AeroSciences Branch 


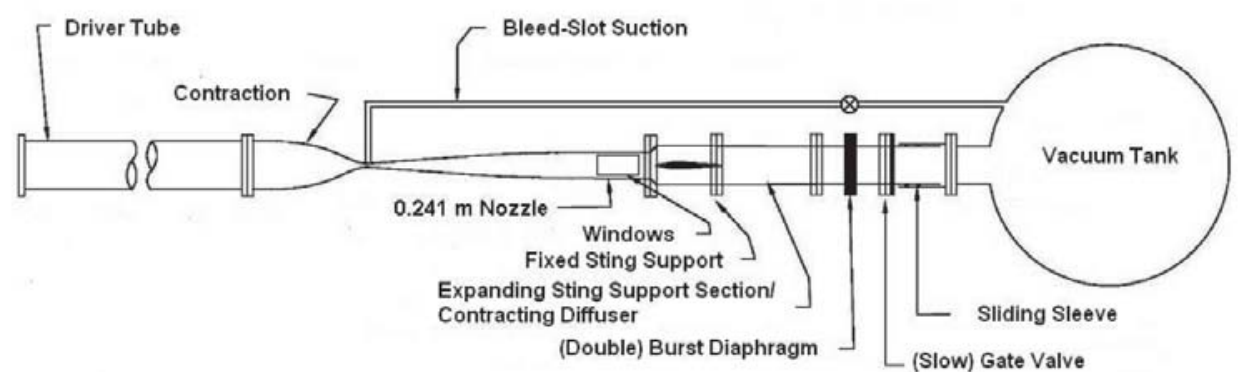

(a) Schematic diagram of the Boeing/AFOSR Mach-6 Quiet Tunnel.

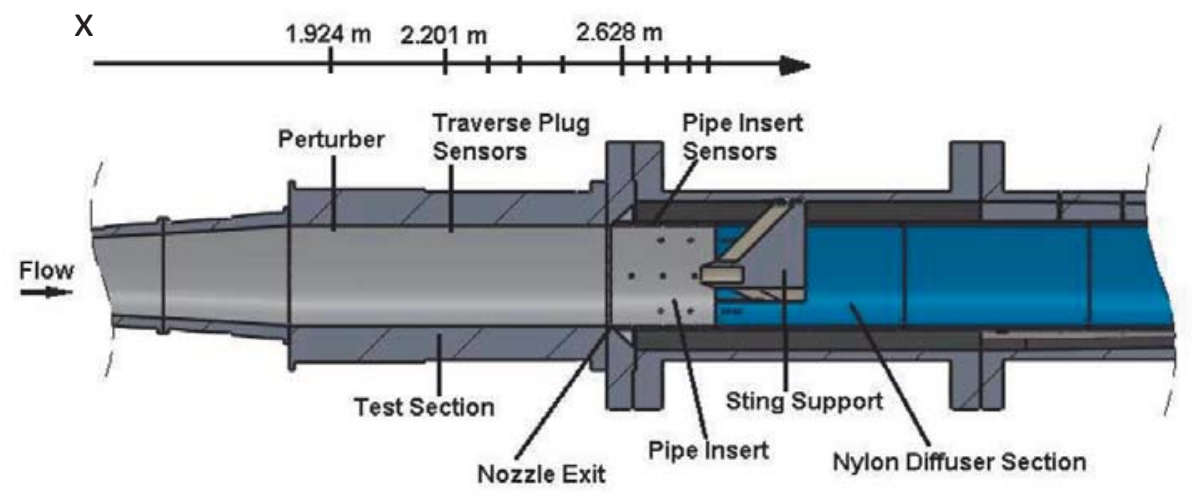

(b) Schematic of experimental setup with perturber and sensor locations denoted on the x-axis.

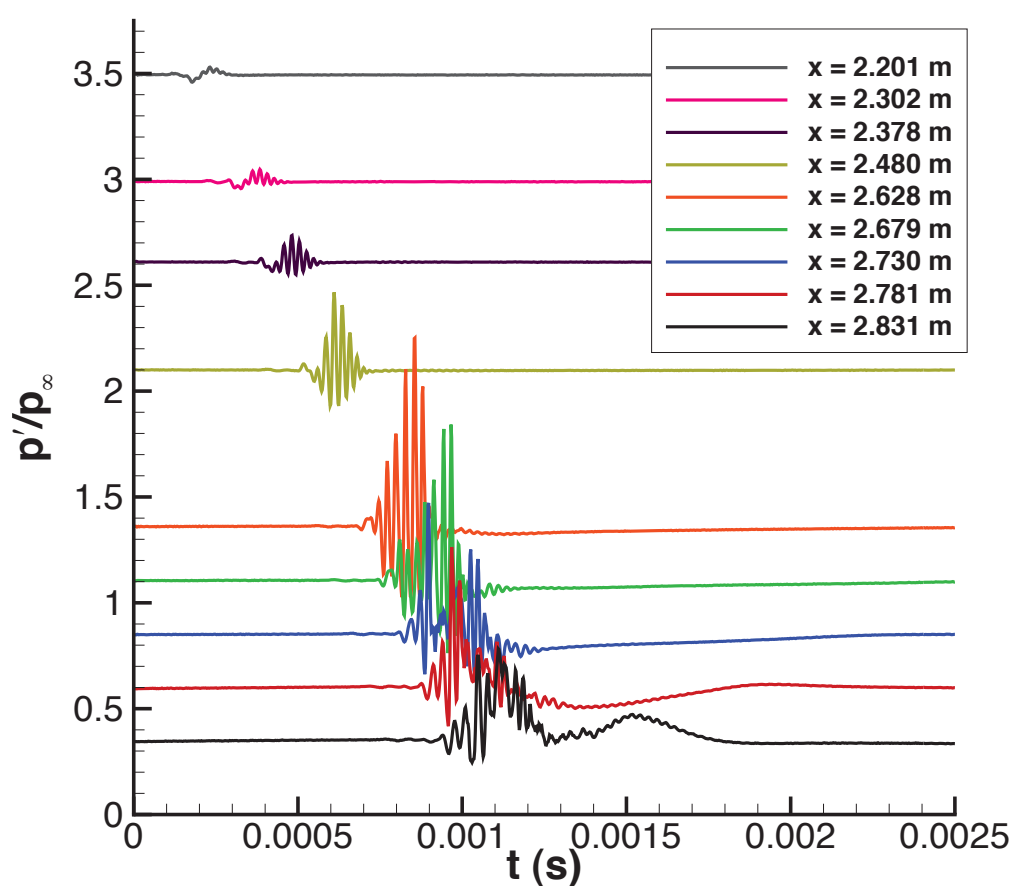

(c) Ensemble-averaged disturbances.

Figure 1. Experiment configuration and typical results from Ref. 1 
the use of larger amplitudes than otherwise possible. Recent experimental work ${ }^{3}$ measured characteristics of the forcing and determined that it was of much longer duration and did not normally involve a spark at all. Instead, the electrode is described as a "pulsed glow perturber." The longer duration reduces the required amplitude of the forcing and results in a much more benign initial transient.

The next section of this paper describes the governing equations and the DG discretization method used to solve them. This is followed by a description of the computational domain and the forcing function used to model the pulsed glow perturber. Finally, results of three-dimensional simulations are presented and compared to both experimental results and to linear stability analysis.

\section{Governing Equations and DG Formulation}

The nondimensional compressible Navier-Stokes equations for a perfect gas in conservation form, using tensor index notation are:

$$
\begin{aligned}
\frac{\partial \rho}{\partial t} & +\frac{\partial\left(\rho u_{j}\right)}{\partial x_{j}}=0 \\
\frac{\partial \rho e}{\partial t} & +\frac{\partial\left(h u_{j}-u_{i} \tau_{i, j}+q_{j}\right)}{\partial x_{j}}=0, \\
\frac{\partial \rho u_{i}}{\partial t} & +\frac{\partial\left(\rho u_{i} u_{j}+\delta_{i, j} P-\tau_{i, j}\right)}{\partial x_{j}}=0, \quad i=1,2,3
\end{aligned}
$$

where $\rho$ is the density, $e$ is the internal energy per unit mass, $P$ is the pressure, $u_{i}$ is the component of velocity in the Cartesian coordinate direction $x_{i}$, and $\delta_{i, j}$ is the Kronecker delta. The quantities $h, q_{i}$, and $\tau_{i, j}$ are the enthalpy, heat flux, and shear stress terms, respectively; and are given by:

$$
\begin{gathered}
h=\rho e+P \\
q_{i}=-\frac{\gamma}{\gamma-1} \frac{1}{\operatorname{Pr}} \frac{\mu}{R e_{r}} \frac{\partial T}{\partial x_{i}} \\
\tau_{i, j}=\frac{\mu}{R e_{r}}\left(\frac{\partial u_{i}}{\partial x_{j}}+\frac{\partial u_{j}}{\partial x_{j}}-\delta_{i, j} \frac{2}{3} \frac{\partial u_{n}}{\partial x_{n}}\right),
\end{gathered}
$$

where $\operatorname{Pr}$ is the Prandtl number, and $R e_{r}$ is the Reynolds number based on the reference state of the nondimensionalization, and $T$ is the temperature given by $T=P / \rho=(\gamma-1)\left(e-u_{i} u_{i} / 2\right)$. The length and the thermodynamic variables have been nondimensionalized with respect to a prescribed reference state: $x_{i}=\hat{x}_{i} / \hat{L}_{r}, \rho=\hat{\rho} / \hat{\rho}_{r}, P=\hat{P} / \hat{P}_{r}, T=\hat{T} / \hat{T}_{r}$, and $\mu=\hat{\mu} / \hat{\mu}_{r}$ where $\left({ }^{\wedge}\right)$ denotes dimensional quantities, and the subscript $r$ denotes the reference state. Other variables are normalized with respect to derived reference states as follows: $u=\hat{u} / \hat{u}_{r}, t=\hat{t} /\left(\hat{L}_{r} / \hat{u}_{r}\right), e=\hat{e}_{r} / \hat{u}_{r}^{2}$, and $R e_{r} \equiv \hat{\rho}_{r} \hat{u}_{r} \hat{L}_{r} / \hat{\mu}_{r}$ where $\hat{u}_{r}=\sqrt{\hat{P}_{r} / \hat{\rho}_{r}}$. Sutherland's formula is used to evaluate both $\hat{\mu}_{r}$ as a function of $\hat{T}_{r}$ and $\mu$ as a function of $T$.

DG is applied to each of Eqs. 1-3 in essentially the same manner, however, the inviscid and viscous terms are treated differently. To facilitate the following discussion, each equation is cast in the general form:

$$
\frac{\partial \tilde{U}}{\partial t}+\nabla \cdot\left(\tilde{\mathbf{F}}_{i}-\tilde{\mathbf{F}}_{v}\right)=0
$$

where $\tilde{U}$ denotes either $\rho$, $\rho$, or $\rho u_{i}$, and $\tilde{\mathbf{F}}_{i}$ and $\tilde{\mathbf{F}}_{v}$ denote the inviscid and viscous contributions to the flux. Equation (4) is transformed to a local computational coordinate system for each discrete element. Let $(\xi, \eta, \zeta)$ denote the local coordinate system with Jacobian $J \equiv \partial\left(x_{1}, x_{2}, x_{3}\right) / \partial(\xi, \eta, \zeta)$. Equation (4) has the same form in the local coordinates:

$$
\frac{\partial U}{\partial t}+\nabla \cdot\left(\mathbf{F}_{i}-\mathbf{F}_{v}\right)=0
$$

but with $U=\tilde{U}|J|, F_{i}=J^{-1} \tilde{\mathbf{F}}_{i}|J|$, and $F_{v}=J^{-1} \tilde{\mathbf{F}}_{v}|J|$. 


\section{A. DG Discretization}

The implementation of DG used in this study follows the quadrature-free form described in Refs. 4,6,7, and is described only briefly here. The computational domain is subdivided into nonoverlapping elements that cover the domain. The DG discretization is formulated locally in each element in a similar manner. The solution within each element is approximated as an expansion in a local basis set $\left\{b_{n}\right\}$, usually polynomials of degree $\leq p$,

$$
U=\sum_{n=0}^{N_{p}} b_{n} u_{n}
$$

where $N_{p}$ denotes the number of terms in the basis set of degree $p$. The current work uses three-dimensional monomials of the form $\xi^{i} \eta^{j} \zeta^{k}$ for all $(i, j, k)$ triplets satisfying $0 \leq i+j+k \leq p$. However, both the formulation and the simulation code readily apply to lower dimensional formulations simply by constraining the basis to lower dimensions. The number of unknowns in each element is the number of physical variables times the size of the basis set, $N_{p}$. An equal number of equations governing these unknowns is derived by multiplying the governing equations by each member of the basis set, and integrating over the element. The integrals of the flux terms are integrated by parts to obtain the following weak form:

$$
\int_{\Omega} b_{n} \frac{\partial U}{\partial t} d \Omega-\int_{\Omega} \nabla b_{n} \cdot\left(\mathbf{F}_{i}-\mathbf{F}_{v}\right) d \Omega+\int_{\partial \Omega} b_{n}\left(\mathbf{F}_{i}-\mathbf{F}_{v}\right) \cdot \mathbf{n} d s=0 \quad \forall k: 0 \leq k \leq N_{p},
$$

where $\Omega$ denotes the element, $\partial \Omega$ denotes the element boundary, and $\mathbf{n}$ denotes the outward unit normal.

Following the quadrature-free formulation, ${ }^{6}$ the fluxes are expanded in a similar manner:

$$
\mathbf{F}_{i}=\sum_{k=0}^{M} b_{n} \mathbf{f}_{i, k} \quad \text { and } \quad \mathbf{F}_{v}=\sum_{k=0}^{M} b_{n} \mathbf{f}_{v, k} .
$$

The degree of the flux expansions is allowed to be higher than that of the solution with the result that $M \geq N_{p}$. The element boundary integral is evaluated piecewise on discrete segments or edges, $\partial \Omega_{j}$, where each edge is shared with a neighboring element. The two neighboring elements on either side of an edge segment have independent local approximations for the solution and fluxes. To resolve this ambiguity, the fluxes in the edge integrals are replaced by numerical fluxes that are a function of the solutions in both neighboring elements. The numerical edge fluxes are represented in terms of a lower dimensional edge basis, $\bar{b}_{k, j}$ :

$$
\left.\mathbf{F}_{i} \cdot \mathbf{n}\right|_{\partial \Omega_{j}} \Longrightarrow \widehat{F}_{i}=\sum_{k=0}^{\bar{M}} \bar{b}_{k, j} \widehat{f}_{i, k, j} \quad \text { and }\left.\quad \mathbf{F}_{v} \cdot \mathbf{n}\right|_{\partial \Omega_{j}} \Longrightarrow \widehat{F}_{v}=\sum_{k=0}^{\bar{M}} \bar{b}_{k, j} \widehat{f}_{v, k, j}
$$

where $\partial \Omega_{j}$ denotes an individual edge and $\bar{M}$ denotes the number of terms in the edge basis.

The inviscid edge flux is modeled by an approximate Riemann flux. The local Lax-Friedrichs flux is simple and inexpensive to implement, and has worked well with DG for smooth flows. ${ }^{4,9}$ However, the HLL flux ${ }^{10}$ has been shown ${ }^{5}$ to be more robust accurate for flows with shock waves, and is used in all simulations presented here.

The solution gradients required for the viscous terms are evaluated using the DG methodology in a similar manner. Let

$$
\boldsymbol{\sigma}_{w}=\sum_{k=0}^{N_{p}} b_{n} \boldsymbol{\sigma}_{n} \equiv \nabla w
$$

where $w$ denotes either $u_{i}$ or $T$. It immediately follows that

$$
\int_{\Omega} b_{n} \boldsymbol{\sigma}_{w} d \Omega-\int_{\Omega} \nabla b_{n} w d \Omega+\int_{\partial \Omega} b_{n} w \mathbf{n} d s=0
$$

As before, the multi-valued edge flux is replaced by a numerical flux, $\left.w \mathbf{n}\right|_{\partial \Omega}=\widehat{w \mathbf{n}}$. Both $\widehat{F}_{v}$ and $\widehat{w \mathbf{n}}$ are evaluated as described in Ref. 4.

Because all of the fluxes are represented as expansions in the basis set, the integrations can be performed directly to produce a matrix equation of the form:

$$
\mathbf{M}\left[\frac{\partial u_{n}}{\partial t}\right]+\mathbf{V} \cdot\left[\mathbf{f}_{i, k}-\mathbf{f}_{v, k}\right]+\sum_{j} \mathbf{B}_{j}\left[\widehat{\mathbf{f}}_{i, k, j}-\widehat{\mathbf{f}}_{v, k, j}\right]=0
$$


where $\mathbf{M}, \mathbf{V}$, and $\mathbf{B}$ are derived in Ref. 6 along with further details of the quadrature-free formulation.

Reference 5 investigated a number of implementation details and variants of DG that influence the accuracy and robustness of the method for flows with shock waves. In most cases, these variants improve the accuracy of DG when shocks are not present, However, the exception is the strategy of augmenting the viscosity in the neighborhood of the shock wave to improve the robustness of the method. While the earlier work demonstrated that the technique did indeed improve robustness, it also revealed some undesirable side effects. In particular, for the preliminary axisymmetric simulations of the current flow, the forcing could be increased by an order of magnitude over what was possible with out applying the technique. However, shock sensors typically used by the approach would also be triggered by the disturbance itself, locally increasing the viscosity and potentially corrupting the simulation. Due to the developmental nature of the augmented viscosity technique, it was decided not use the technique in the $3 \mathrm{D}$ simulations presented here. While this choice did restrict the maximum forcing amplitude that could be run, the more gentle nature of the longtimescale forcing (Eq. 9 below) allowed 3D simulations with sufficiently large amplitudes to run successfully.

\section{Simulation}

Time accurate unsteady simulations were performed starting from a shock-free steady flow solution described in Ref. 11. The initial residuals were subtracted from all following values to eliminate small transients that could arise due to differences in the discretization schemes and grid resolution. The Reynolds number is $9.55 \times 10^{6} / \mathrm{m}$. The discretization is a fourth-order DG on tetrahedral elements based on the quadrature-free formulation. ${ }^{6,12}$ Time advancement uses the explicit third-order TVD-RK scheme of Shu. ${ }^{13}$

\section{A. Computational Domain}

The computational domain, shown in Fig. 2, is defined by the tunnel wall, two nearly conical sections, two ramp sections, and two $\mathrm{x}=$ constant planes for the inflow and outflow boundaries. The conical and ramp

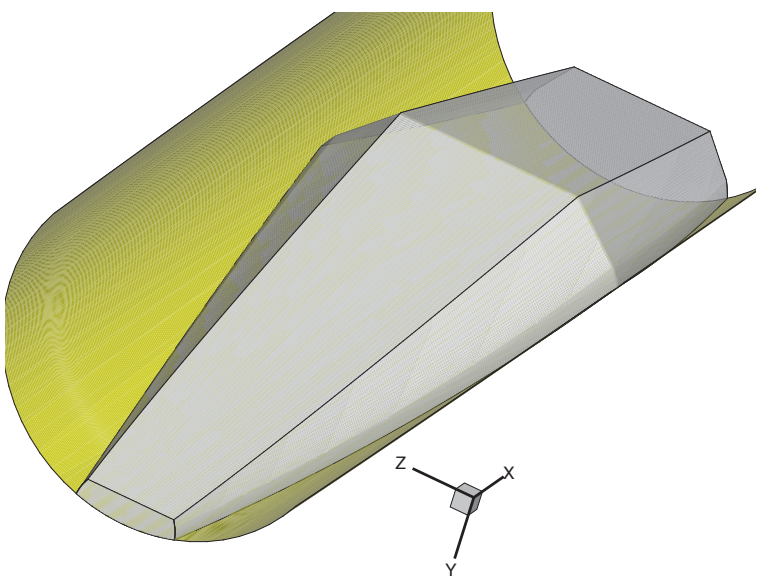

(a) Domain boundaries

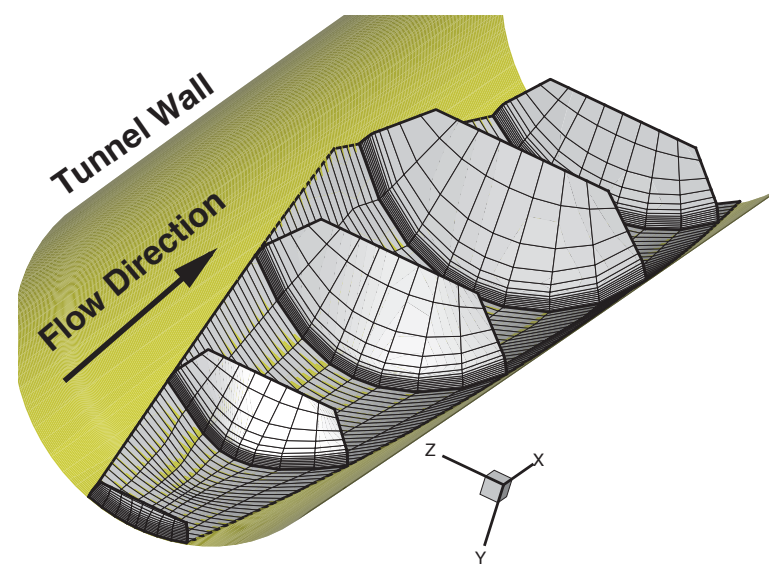

(b) Grid cross-section on tunnel wall and several cross-flow sections; showing every fourth line.

Figure 2. Computational domain and grid.

sections are inclined steeper than the local Mach cones expected in the Mach 6 freestream such that these inflow and outflow boundaries are not of a mixed type (i.e. characteristic directions at boundary points are either all inbound or all outbound). The underlying grid is a curvilinear, single-block structured grid in which each quad-element is subdivided into five tetrahedrons. Grid stretching is applied in the wall-normal direction to place approximately half of the points within the boundary layer. Within the boundary layer, grid points are clustered toward the forcing region and toward the centerline. In the streamwise direction, the domain extends from $x=1.85 \mathrm{~m}$ to $x=2.584 \mathrm{~m}$, for most of the results show here. The downstream extent of the domain in the current simulations is limited by the domain of the steady starting flow solution taken from Ref. 11. A typical grid size of $345 \times 54 \times 32$, with five tetrahedrons per quad and 20 degrees 
of freedom per polynomial of degree three yields approximately 60 million degrees of freedom per equation. The accuracy of simulation results was evaluated by comparing them to results on a coarser grid for isolated cases, and is judged to be more then adequate for the current flow. These comparisons will be shown in a later section.

\section{B. Modeling Effect of Electrode}

The primary effect of the electrode is to inject energy into the flow field. This effect is modeled numerically by adding a source term of the form $A_{0} S(x, y, z) T(t)$ to the energy equation (right hand side of Eq. 2). Little was know at the outset of this work about the forcing produced by the experimental apparatus; and in the preliminary two-dimensional axisymmetric simulations, ${ }^{5}$ the source term was modeled as simple pulse:

$$
\begin{aligned}
S(x, y, z) & =[(1+\cos (\pi \hat{r})) / 2]^{2} \\
T(t) & =\sin (\pi \hat{t})^{4}
\end{aligned}
$$

where

$$
\begin{aligned}
\hat{r} & =\min \left(1, \sqrt{\left(x-x_{s}\right)^{2}+\left(\left(y-y_{s}\right) / \alpha\right)^{2}+\left(\left(z-z_{s}\right) / \beta\right)^{2}} / r_{f}\right), \\
\hat{t} & =\min \left(1, t / t_{d}\right),
\end{aligned}
$$

$t_{d}$ is the duration of the forcing, $\hat{r}$ is a directionally scaled distance from the center of the source, $r_{f}$ is the radius of the forcing region, and $A_{0}$ is the forcing amplitude. The origin of the forcing is at $(x, y, z)_{s}=$ $(1.924,0.117,0.0)$. The radius of the forcing region was set to $0.00127 \mathrm{~m}$, which approximately equals the electrode gap. To elongate the forcing region in the streamwise direction, $\alpha$ and $\beta$ are set to $1 / 2$. The duration was set to $2 \times 10^{-5}$ s by matching the period of the disturbance at $x=2.201$. The amplitude $A_{0}$ was tuned to match the disturbance amplitude at multiple probe locations and typically ranged between $5 \times 10^{4}$ to $6 \times 10^{5}$.

Recent experimental work ${ }^{3}$ suggests that the duration of the forcing is much longer than originally assumed, and the following model for $T(t)$ is used in the 3D simulations.

$$
T(t)=16\left[\sin (\pi \hat{t})+\sin (2 \pi \hat{t})^{2} / 2\right]\left[\left(1-A_{1}\right)+A_{1} \cos \left(\pi\left(2 t-t_{d} / 3\right) / t_{h}\right)\right] / 27 .
$$

The primary forcing is now asymmetric, and the new parameters, $A_{1}$ and $t_{h}$ denote the amplitude and period, respectively, of a short-period component that is matched to the period of the disturbance. Forcing parameters $t_{d}=1.624 \times 10^{-4}(\mathrm{~s}), t_{h}=2.17 \times 10^{-5}(\mathrm{~s})$ and $r_{f}=0.01 \mathrm{~m}$ are used in the simulations presented below; $A_{0}$ and $A_{1}$ are varied to approximately match the character of experimental results at $x=2.055$ and the period of the disturbance at $x=2.201$.

\section{Axisymmetric Simulations}

Axisymmetric simulations were used to both size the forcing parameters and to evaluate mesh size requirements. Figures 3(a) and (b) illustrate the two types of forcing functions and the disturbances they produce. Pressure data at each probe are normalized by their local initial (undisturbed) values, $P_{0}$. The simple $\sin (x)^{4}$ type of forcing produces a symmetric wave packet at a targeted frequency. However, the experimental measurements indicated that, at this location, the disturbance should be small and superimposed onto a long-period transient. The more complex asymmetric forcing given by Eq. 9 has this desired characteristic. The amplitude of the long-time transient seen in the experiment is still much larger than that of the simulation. However, further effort to reverse engineer the simulation forcing is beyond the scope of this work.

Figures 4(a) and (b) show the results at two probe locations for a range of mesh sizes. Results are grid converged on the $320 \times 75$ grid, and are acceptable on the $213 \times 50$ mesh. Most 3D simulations are performed on a grid similar to the $320 \times 752 \mathrm{D}$ grid. However, the number of points in the wall normal direction are decreased in the $3 \mathrm{D}$ case by increasing the stretching outside the boundary layer, and by decreasing the distance to the outer boundary. The number of points in the stream wise direction was increased in the 3D grid, relative to the $2 \mathrm{D}$ grid, to provide better resolution near the forcing. 


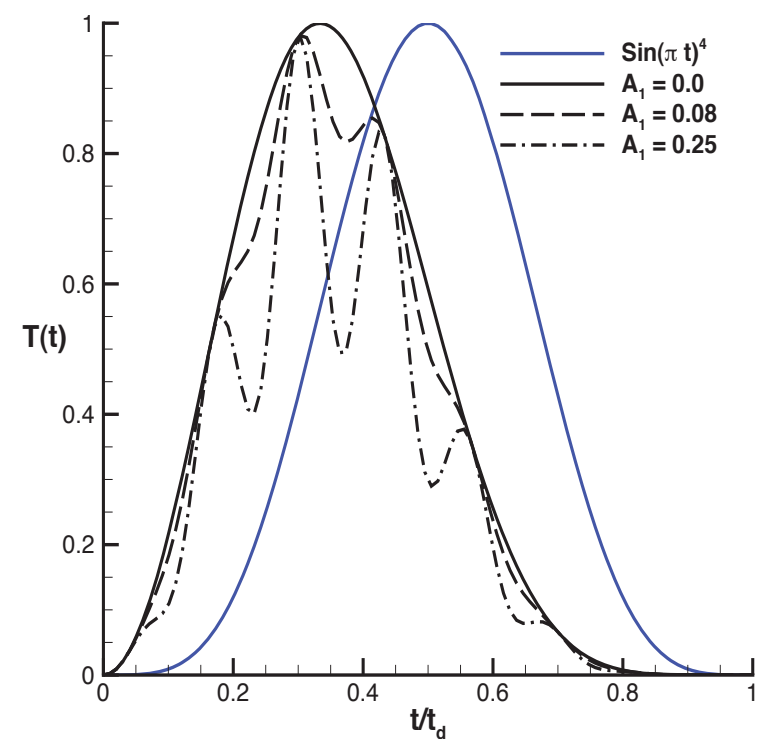

(a) Temporal forcing function $T(t)$ : blue line indicates the simple symmetric pulse, black lines indicated the complex asymmetric pulse given by Eq. 9 for given values of $A_{1}$.

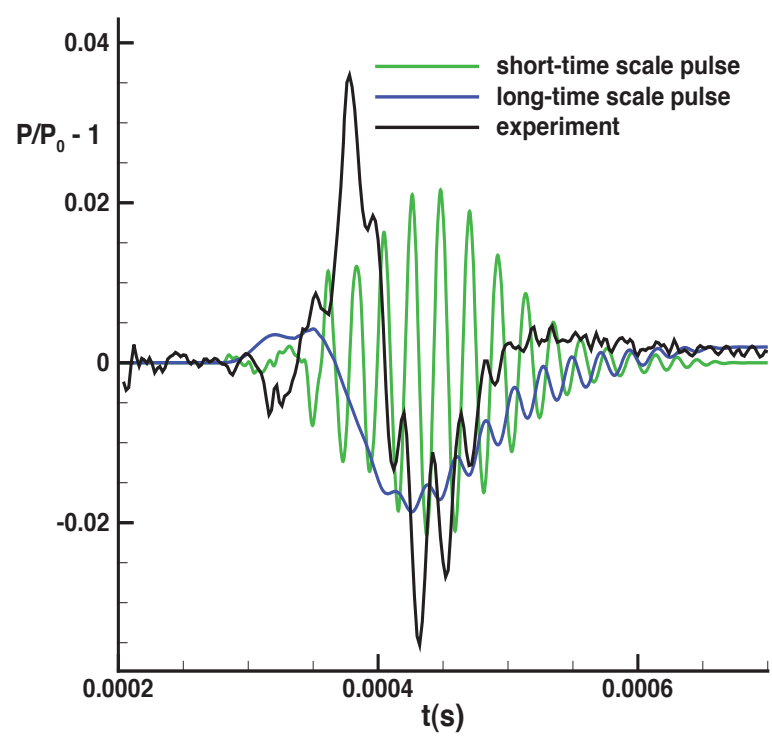

(b) Disturbance produced by the two types of forcing at $x=$ 2.201 .

Figure 3. Forcing functions and disturbances produced.

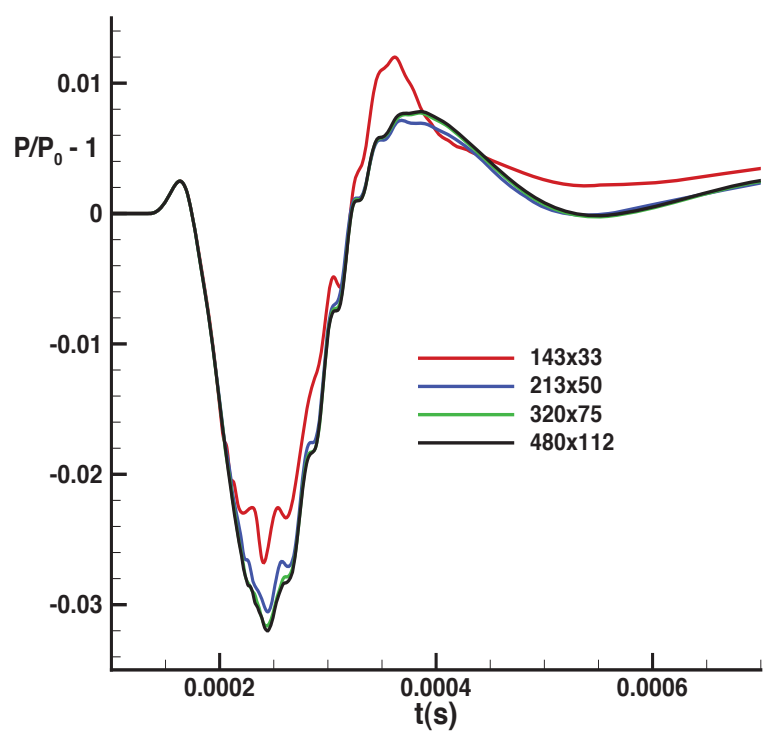

(a) Probe location at $x=2.055$.

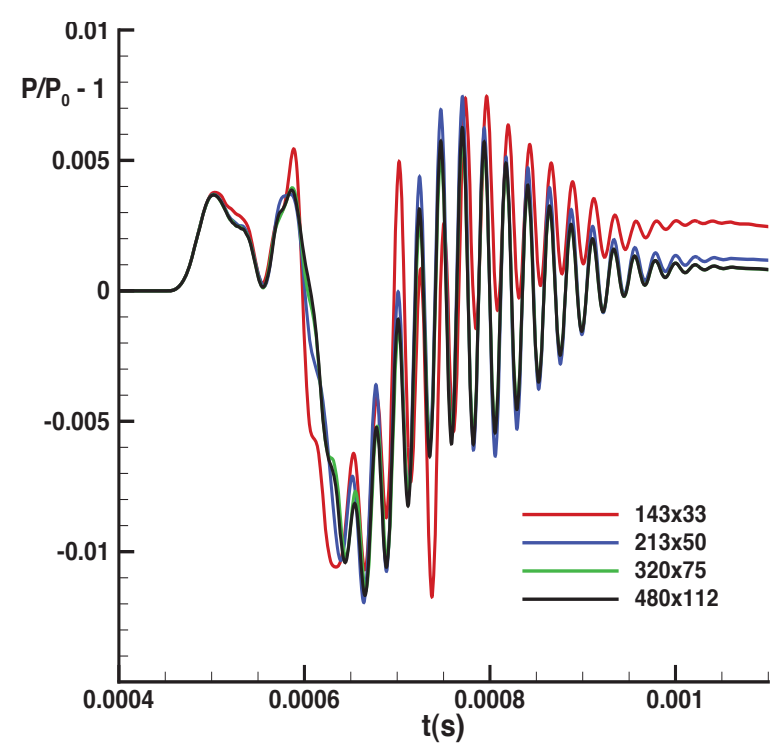

(b) Probe location at $x=2.480$.

Figure 4. Mesh refinement study at two probe locations. 


\section{Three-Dimensional Simulation Results}

The experimental data consist of the time history of pressure measured by probes on the tunnel wall at several locations downstream of the pulse glow perturber. The data are post-processed as described in Ref. 1. Only 5 of the probe locations fall within the computational domain of the simulation. Similar data were collected during the simulations at each of these locations, as well as at an additional 478 points. The forcing parameters, $A_{0}$ and $A_{1}$ were tuned to approximately match the disturbance amplitude observed in the experiment at $x=2.201 \mathrm{~m}$. Figures 5 (a) and (b) show the experimental and simulation results for the common set of probe locations with $A_{0}=3,710$ and $A_{1}=0.078167$. Qualitatively, the principal period of the disturbance and its group velocity agree well with the experiment. In the experiment, the wave packet has a short period component superimposed over a much broader transient. The simulated results have a similar feature, but it is much less pronounced.

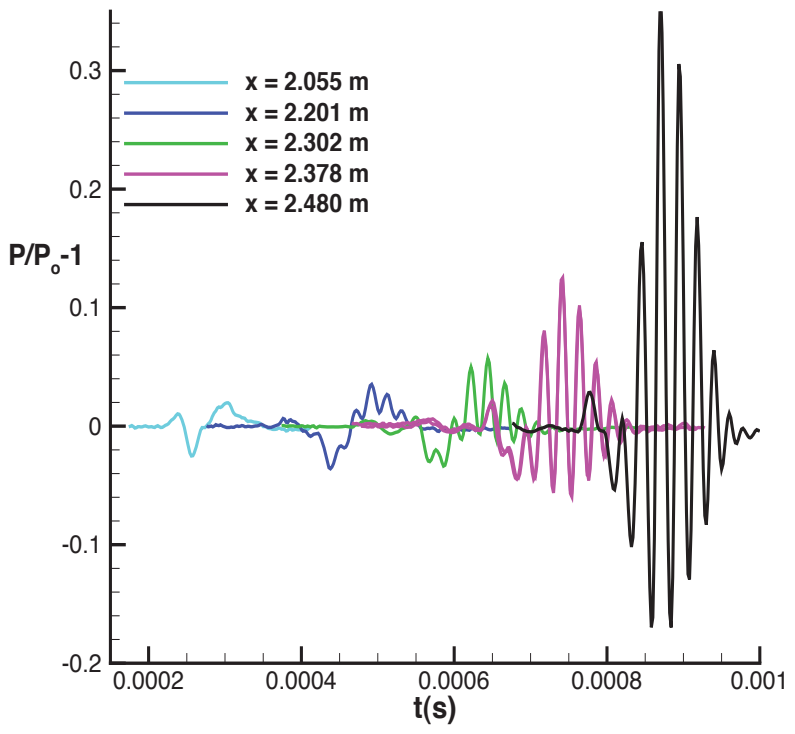

(a) Experimental results.

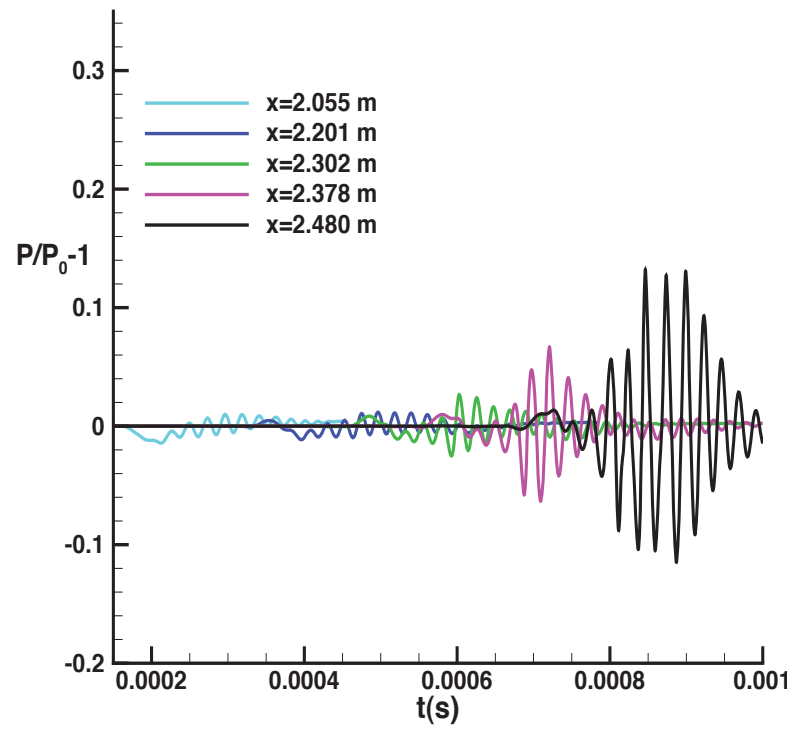

(b) Simulation results with $A_{0}=3,710, A_{1}=0.078$.

Figure 5. Time history of pressure at probe locations common to both data sets.

To precisely distinguish between the two components, the data are further decomposed into a longtimescale transient and a short-timescale symmetric disturbance. Figures 6 (a) and (b) illustrate the results of this process for the experimental data at the probe location $x=2.480$. The decomposition method is a heuristic procedure based on identifying extrema. The method works well when the mode scales are well separated but can locally fail under some well understood circumstances. For the current purposes, the method can be reliably used to precisely quantify features of the symmetric disturbance such as its period and growth rate. The decomposition is constructed by creating envelopes defined by cubic fits through local maximums and minimums. The long-timescale transient is defined as the average of the upper and lower envelopes. The short-timescale symmetric disturbance is defined as the difference between the actual signal and the long-timescale component. Subtracting the long-timescale from the upper and lower envelopes yields the envelope of the short-timescale symmetric disturbance. In the following discussion, the disturbance amplitude $A_{d}$ is defined to be the maximum of the disturbance envelope, $t_{\text {peak }}$ is the time at which the disturbance envelope reaches its peak, and the principal period $t_{\text {period }}$ is the peak-to-peak time interval averaged over the three cycles nearest the peak disturbance. Growth rates per meter, $g$, are computed from adjacent probes located at $x_{i}$ and $x_{i-1}$ as:

$$
g=\ln \left(A_{d, i} / A_{d, i-1}\right) /\left(x_{i}-x_{i-1}\right)
$$

Applying this procedure to all data samples gives the results shown in Fig. 7. The amplitude of the long-timescale transient is larger than the disturbance for the first three probe locations of the experimental results. At the most upstream probe location, the disturbance is nearly undetectable in the full pressure trace, and the decomposition method is not reliable for this probe. In the simulation results, the long-scale 


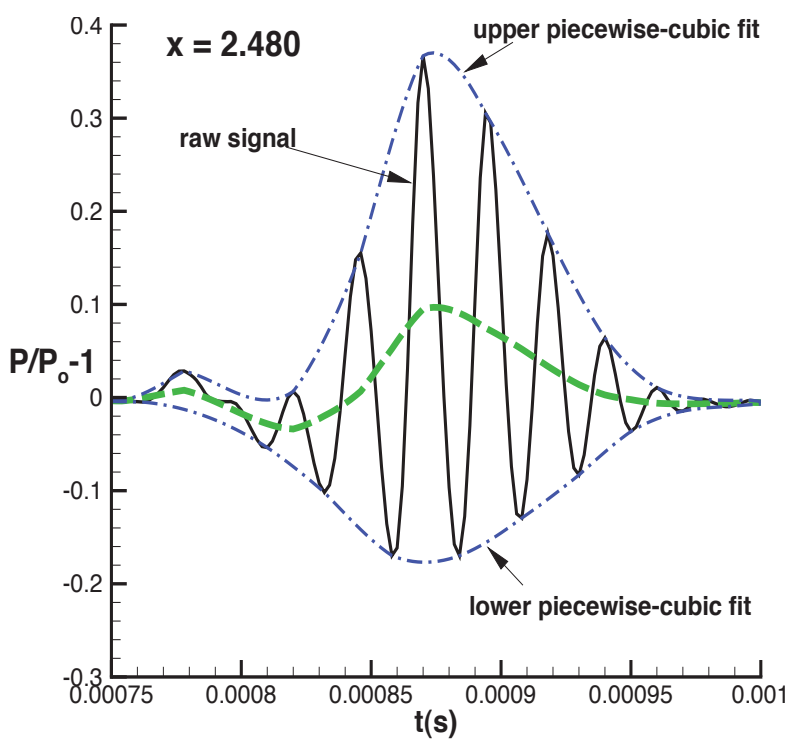

(a) Full pressure trace.

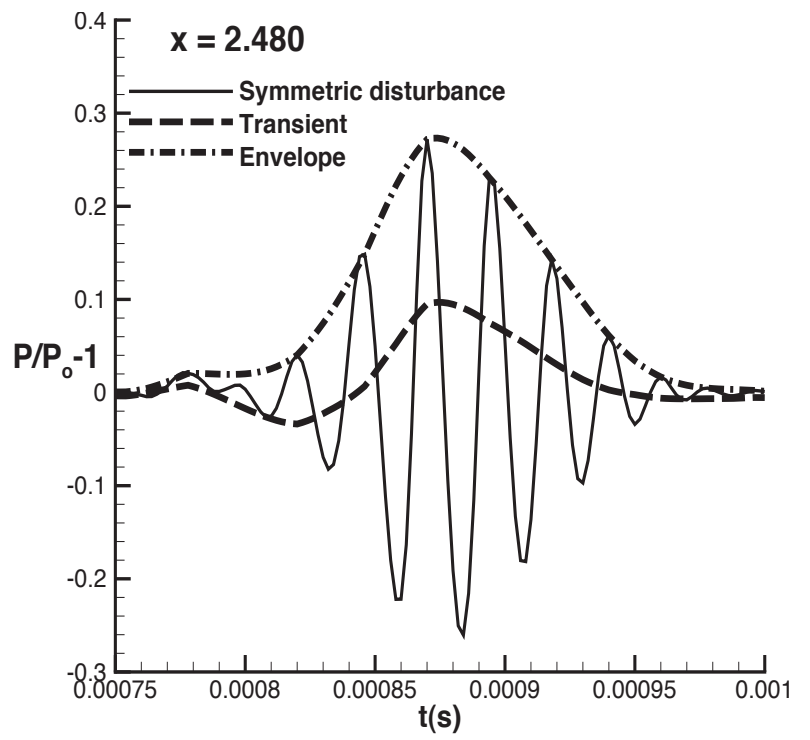

(b) Long- and short-timescale components.

Figure 6. Decomposition of data into long-timescale transient and short-timescale symmetric disturbance.

transient amplitude is comparable to that of the disturbance only at the most upstream probe, and the decomposition procedure is judged to be reliable at all probe locations.

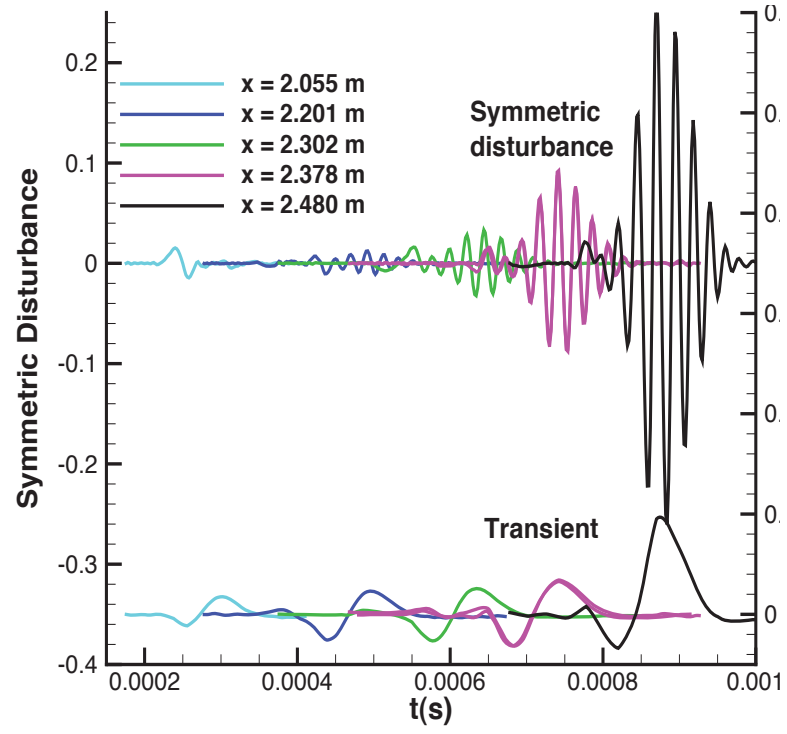

(a) Experimental results.

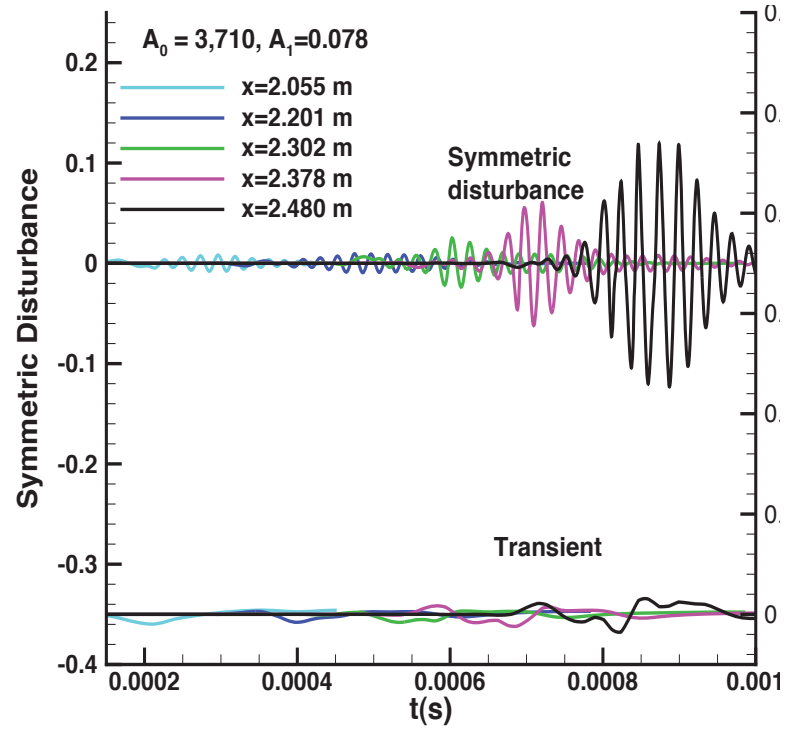

(b) Simulation results with $A_{0}=3,710, A_{1}=0.078$.

Figure 7. Time history of long-timescale transient and short-timescale symmetric disturbance at probe locations that are common to both sets.

With the disturbance component isolated, disturbance features such as amplitude, growth rate, group velocity, and period can be precisely determined, and are presented in Figs. 8 and 9. The simulation data include additional probe locations between those of the experiment; and the experimental data include additional probe locations that are downstream of the simulation domain. The figures show results for several combinations of forcing amplitude. However the simulation results agree very well with the experimental result only for the case with the largest forcing amplitude (black curve).

It was expected at the outset that for any given value of long-mode forcing $A_{0}$, the short-mode forcing parameter $A_{1}$ could be tuned to match the experimental results; however, such was not the case. Figures 8 and 9 also show results from two additional simulations in which the forcing amplitude of the short 


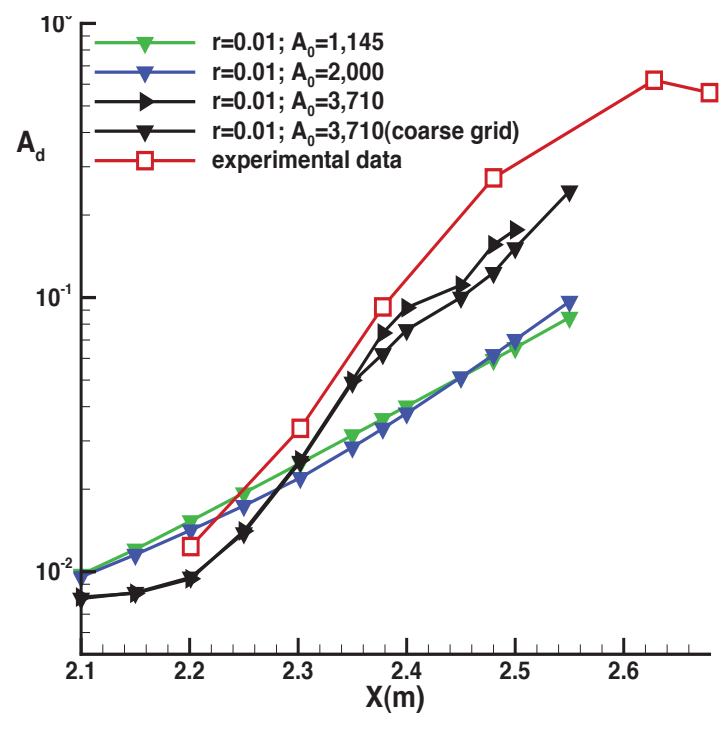

(a) Amplitude of disturbance.

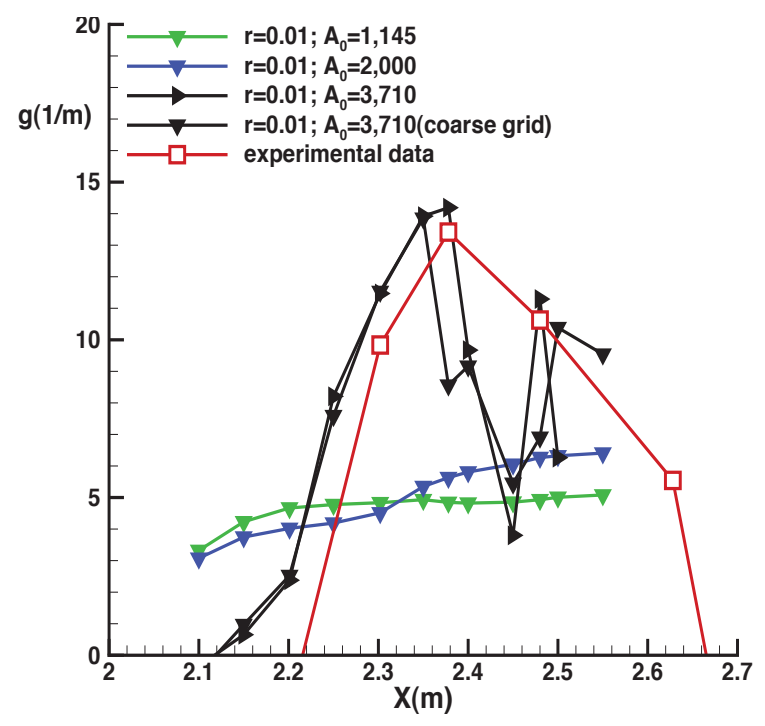

(b) Growth rate (per meter) of disturbance.

Figure 8. Amplitude and growth rate of the symmetric disturbance.

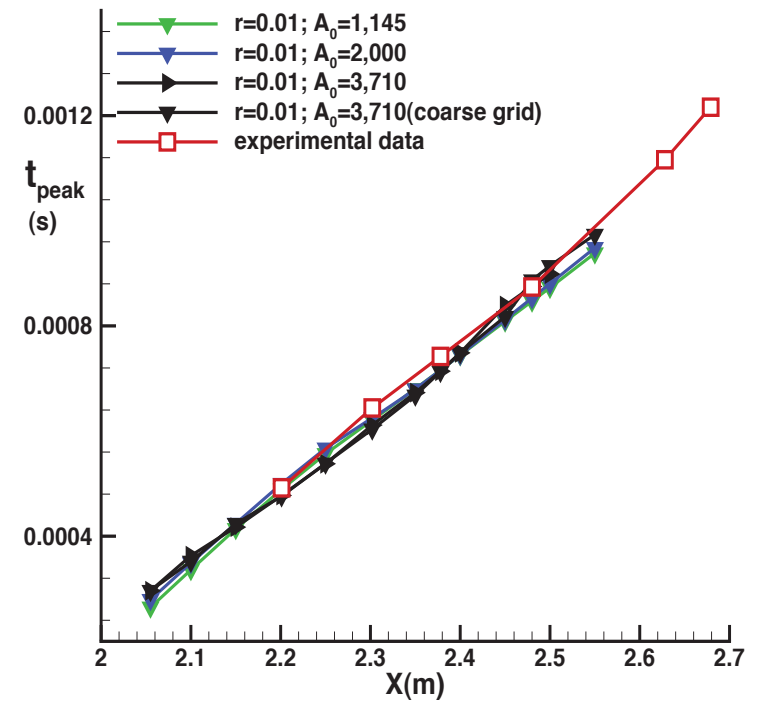

(a) Time of peak disturbance.

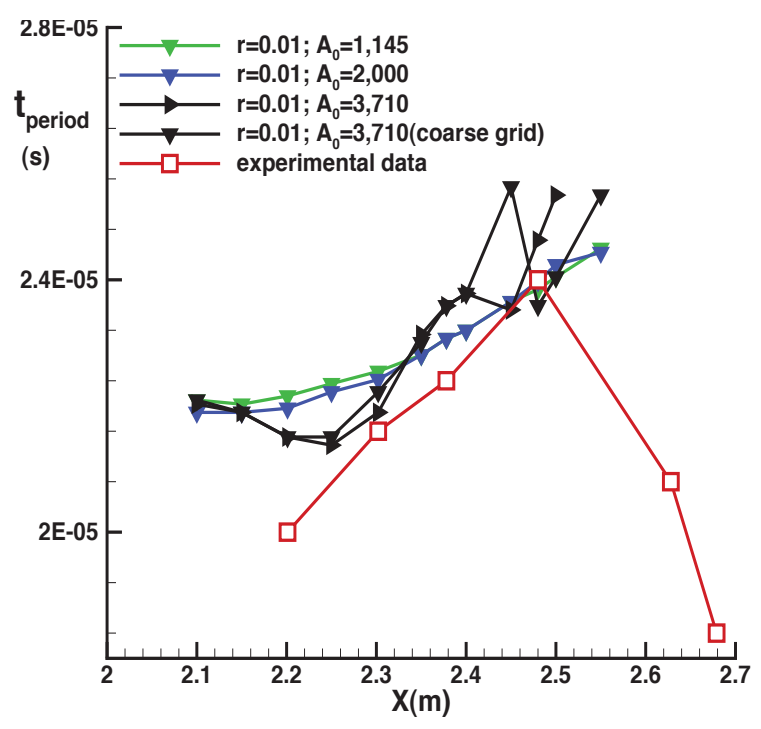

(b) Period of disturbance.

Figure 9. Time of peak disturbance amplitude and period of disturbance at peak. 
mode $\left(A_{0} A_{1}\right)$ is held fixed, while the amplitude of the long mode $\left(A_{0}\left(1-A_{1}\right)\right)$ is reduced by half (blue curve), and half again (green curve). In these cases with lower long-mode forcing, the disturbance amplitude is matched at $x=2.055$, but the growth rate is nearly constant through the computational domain, and is lower than the peak values observed in both the experiment and in the simulation with higher long-mode forcing. Figure 9 (a), which plots the time at which the disturbance amplitude reaches its value peak, $t_{\text {peak }}$, indicates that the speed of the disturbance is not significantly influenced by the level of the long-mode forcing. All simulations give similar values for the disturbance period. However, the simulation with the largest long-mode forcing does shift more towards the experimental values (e.g., lower upstream values that gradually increase in the streamwise direction).

\section{Linear Stability Analysis}

To gain insight into the differences seen in the simulations with the variations the forcing amplitude, a linear stability analysis was performed using the Langley Stability and Transition Code (LASTRAC). ${ }^{14}$ The mean flow input required by LASTRAC was constructed from the same mean flow used in the flow simulations. Fig. 10(a) shows growth rates predicted by LASTRAC for an analysis of second-mode instability wave over a narrow range of frequencies. Each mode has a peak growth rate of about eight, which is much lower than the rates of the experiment, or of the simulation with large forcing amplitude. Also, none of the modes have a sustained growth rate over the entire spatial range. This suggest, that the forcing in both the experiment and in the simulation must create a disturbance with a rich complement of modes. Integrating the growth rates of each mode gives the disturbance amplitudes shown in Fig. 10(b). The thick dashed line is the result of averaging the amplitudes of all modes that are growing in the region of interest. The growth rate of the average is about four, shown as the thick dash line in Fig. 10a and agrees well with the simulation results with low amplitude forcing.

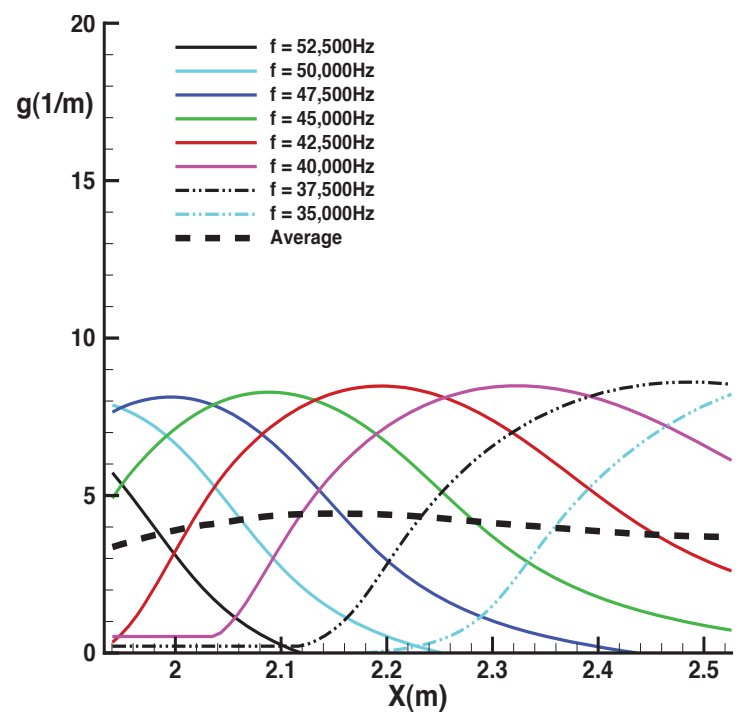

(a) Growth rates.

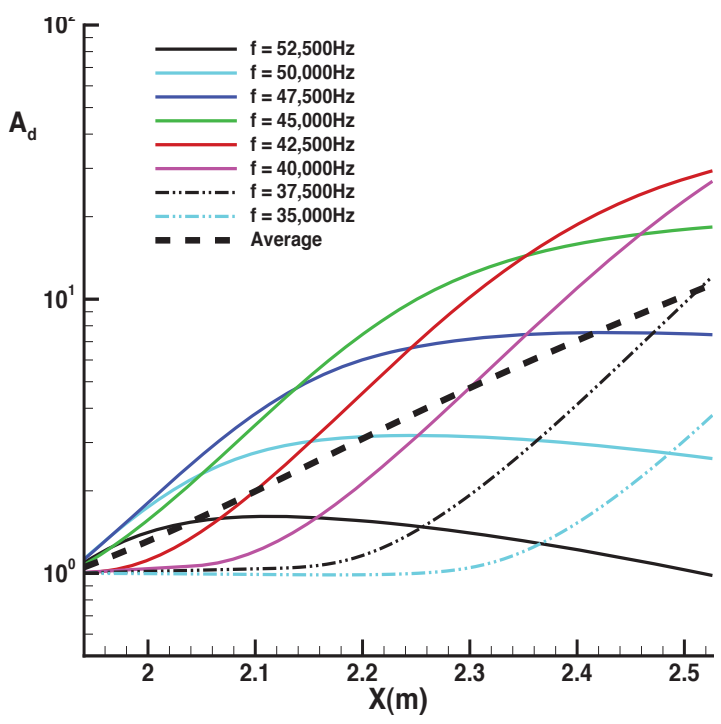

(b) Integrated amplitudes.

Figure 10. Results from linear stability analysis on a uniform spectrum.

It is possible to expand the numerical forcing function to reveal all of the modes it produces. Expanding 
a simplified version of trigonometric functions of Eq. 9 gives:

$$
\begin{gathered}
{\left[\sin \left(\pi t / t_{d}\right)+\frac{1}{2} \sin \left(2 \pi t / t_{d}\right)^{2}\right]\left[\left(1-A_{1}\right)+A_{1} \cos \left(2 \pi t / t_{h}\right)\right]} \\
=\left(1-A_{1}\right)\left\{5+4\left[\cos \left(\pi t / t_{d}\right)-\cos \left(2 \pi t / t_{d}\right)-\cos \left(3 \pi t / t_{d}\right)\right]-\cos \left(4 \pi t / t_{d}\right)\right\} / 8 \\
+A_{1}\left\{10 \cos \left(2 \pi t / t_{h}\right)-\cos \left(2 \pi t\left(2 / t_{d}-1 / t_{h}\right)\right)-\cos \left(2 \pi t\left(2 / t_{d}+1 / t_{h}\right)\right)\right. \\
+4\left[\cos \left(\pi t\left(1 / t_{d}-2 / t_{h}\right)\right)+\cos \left(\pi t\left(1 / t_{d}+2 / t_{h}\right)\right)\right. \\
-\cos \left(\pi t\left(3 / t_{d}-2 / t_{h}\right)\right)+\cos \left(\pi t\left(3 / t_{d}+2 / t_{h}\right)\right) \\
\left.\left.-\cos \left(\pi t\left(4 / t_{d}-2 / t_{h}\right)\right)+\cos \left(\pi t\left(4 / t_{d}+2 / t_{h}\right)\right)\right]\right\} / 16
\end{gathered}
$$

Evaluating the above using $t_{d}=1.62451 \times 10^{-4} \mathrm{~s}$ and $t_{h}=2.171158 \times 10^{-5} \mathrm{~s}$ reveals that the forcing is exciting the following frequencies: $3,078 \mathrm{~Hz}, 9,233 \mathrm{~Hz}, 6,155 \mathrm{~Hz}, 12,311 \mathrm{~Hz}, 33,745 \mathrm{~Hz}, 36,825 \mathrm{~Hz}, 39,903 \mathrm{~Hz}, 42,980 \mathrm{~Hz}$, $46,058 \mathrm{~Hz}, 49,136 \mathrm{~Hz}, 52,214 \mathrm{~Hz}, 55,292 \mathrm{~Hz}$ and $58,370 \mathrm{~Hz}$. Figure 11 shows the growth rates and integrated amplitudes predicted by LASTRAC for the unstable modes of this list. The thick black lines indicate the results from uniformly averaging the individual modes and of a weighted average using the actual weights suggested by Eq. 10.

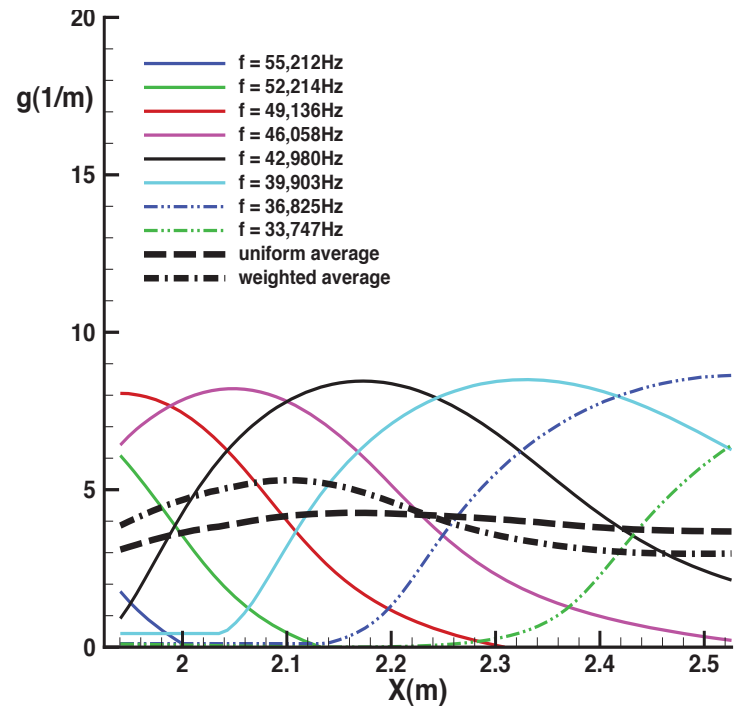

(a) Growth rates.

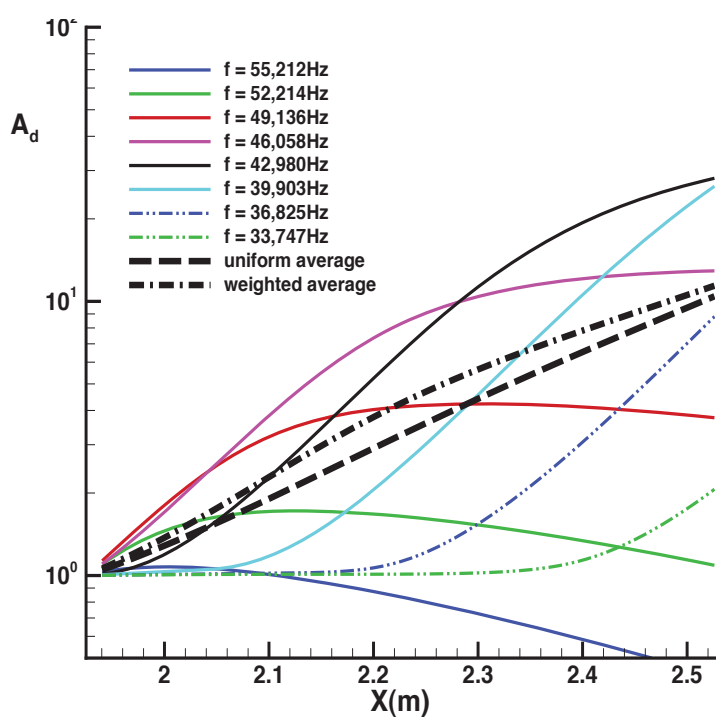

(b) Integrated amplitudes.

Figure 11. Results from linear stability analysis on the spectrum produced by the simulation forcing.

\section{Conclusions}

Simulations of induced disturbances in a laminar Mach 6 boundary layer have been performed using a high-order DG method on an unstructured grid. At a high forcing amplitude, the amplitude, growth rate, group velocity and period of the disturbance agree well with experiments performed in the Boeing/AFOSR Mach-6 Quiet Tunnel at Purdue University. However, the peak growth rates of both cases are three times greater those predicted by the linear stability analysis.

In simulations with low forcing amplitude, the growth rate of the disturbance is lower and nearly constant over the domain. However, the group velocity and period of the disturbance are still similar to that of the experiment and of the simulation with high forcing amplitude. Linear stability analysis predicts that frequencies between $35 \mathrm{kHz}$ to $55 \mathrm{kHz}$ are unstable over some portion of the domain of interest, but that no single mode is unstable over the entire region. Individual modes have peak growth rates of about eight. Both uniform and weighted sums of unstable modes produce growth rates of about four throughout the region, which agrees well with simulations using lower forcing amplitudes.

The high growth rates seen in the experiment and in the simulations with large amplitude forcing are most likely due to nonlinear flow effects. The uneven growth rate seen in the simulation is attributed to the forcing function that populates only a few discrete modes, where as the pulse glow perturber likely induces a continuous spectrum of modes. The sensitivity of the growth rate to the overall forcing amplitude, even 
while holding the amplitude of the short-period forcing mode fixed, suggest that details of the forcing play a critical role in the outcome. Additional measurements to characterize the pulse glow perturber and its effects on the immediate flow field are needed for rigorous CFD validation.

\section{Acknowledgments}

This work was funded by the Hypersonic and Supersonic projects of the Fundamental Aeronautics Program. The author would like to thank Drs. Casper, Beresh, and Schneider for the use of their experimental results and illustrations in this paper. The author would also like to thank Mr. Patrick Greene of UCLA and his team for the use of their mean flow solution. The author would like to acknowledge Dr. Fei Li of NASA Langley Research Center for his assistance in setting up the LASTRAC analysis.

\section{References}

\footnotetext{
${ }^{1}$ Casper, K. M., Beresh, S. J., and Schneider, S. P., "Pressure Fluctuations Beneath Turbulent Spots and Instability Wave Packets in a Hypersonic Boundary Layer," AIAA Paper 2011-372, January 2011.

${ }^{2}$ Casper, K. M., Beresh, S. J., and Schneider, S. P., "Spanwise Growth of a Turbulent Spot Pressure-Fluctuation Field in a Hypersonic Boundary Layer," AIAA Paper 2011-3873, June 2011.

${ }^{3}$ Casper, K. M., Pressure Flucuations Beneath Instability Wave Packets and Turbulent Spots in a Hypersonic Boundary Layer, Ph.D. thesis, Purdue University, Indiana, Aug. 2012.

${ }^{4}$ Atkins, H. L., "Super-convergence of Discontinuous Galerkin Method Applied to the Navier-Stokes Equations," AIAA Paper 2009-3787, June 2009.

${ }^{5}$ Atkins, H. L. and Pampell, A., "Robust and Accurate Shock Capturing Method for High-Order Discontinuous Galerkin Methods," AIAA Paper 2011-3058, June 2011.

${ }^{6}$ Atkins, H. L. and Shu, C. W., "Quadrature-Free Implementation of Discontinuous Galerkin Method for Hyperbolic Equations," AIAA Journal, Vol. 36, No. 5, 1998, pp. 775-782.

${ }^{7}$ Atkins, H. L. and Lockard, D. P., "A High-order Method using Unstructured Grids for Aeroacoustic Analysis of Realistic Aircraft Configurations," AIAA Paper 1999-1945, May 1999.

${ }^{8} \mathrm{Hu}$, F. Q. and Atkins, H. L., "Eigensolution analysis of the discontinuous Galerkin method. Part I: One space dimensions," J. Comput. Phys., Vol. 182, No. 2, 2002, pp. 516-545.

${ }^{9} \mathrm{Hu}, \mathrm{F}$. Q. and Atkins, H. L., "Two-dimensional Wave Analysis of the Discontinuous Galerkin Method with Non-Uniform Grid and Boundary Conditions," AIAA Paper 2002-2514, June 2002.

${ }^{10}$ Harten, A., Lax, P. D., and van Leer, B., "On Upstream Differencing and Godunov-Type Schemes for Hyperbolic Conservation Laws," SIAM Review, Vol. 25, No. 1, 1983, pp. 35-61.

${ }^{11}$ Greene, P. T., Eldredge, J. D., Zhong, X., and Kim, J., "A Numerical Study of Purdue's Mach 6 Tunnel with a Roughness Element," AIAA Paper 2009-174, January 2009.

${ }^{12}$ Atkins, H. L., "Local Analysis of Shock Capturing Using Discontinuous Galerkin Methodology," AIAA Paper 1997-2032, June 1997.

${ }^{13} \mathrm{Shu}$, C.-W., "Total-variation-diminishing time discretizations," SIAM Journal of Scientific and Statistical Computing, Vol. 9, No. 6, 1988, pp. 1073-1084.

${ }^{14}$ Chang, C.-L., "Langley Stability and Transition Analysis Code (LASTRAC) Version 1.2 Users Manual," NASA/TM2004-213233, June 2004.
} 